\title{
ISLA DE CALOR NOCTURNA EN UNA CIUDAD MEDITERRÁNEA DE INTERIOR: INCA (MALLORCA)
}

\author{
Gabriel Alomar-Garau \\ gabriel.alomar@uib.es \\ Cristòfol Rotger Pujadas \\ tofolgeo@gmail.com \\ Marc Aguiló Rodríguez \\ marcaguilo10@gmail.com \\ Neus Lacomba Moreno \\ neuslacomba99@gmail.com \\ Universitat de les Illes Balears, \\ Carretera de Valldemossa, km 7.5, Palma (Illes Balears)
}

\begin{abstract}
Resumen: Se presenta un nuevo estudio del fenómeno de isla de calor urbana en la ciudad mallorquina de Inca, con el objetivo de ampliar y verificar los resultados de los trabajos precedentes. A partir del método de los transectos móviles, se han realizado mediciones en cuatro estaciones del año meteorológico: invierno, otoño, verano y primavera. Los resultados confirman islas de calor nocturnas que manifiestan diferencias de temperatura de hasta $5,7^{\circ} \mathrm{C}$-otoño- entre el sector central de la ciudad y su entorno rural. La actividad antropogénica urbana en Inca, la anchura reducida de ciertas calles, los edificios altos, la escasez de cubierta vegetal y la estabilidad atmosférica, son factores combinados que juegan a favor de este incremento térmico diferencial, aparentemente más acusado en condiciones de frío ambiental que en condiciones de calor. Los resultados son de utilidad para trabajos de planificación urbana con criterios de sostenibilidad ambiental.
\end{abstract}

Palabras clave: Isla de calor urbana, clima urbano, transectos urbanos, Mallorca, Inca. 


\title{
Nocturnal heat island in an inland mediterranean city: Inca (Majorca)
}

\begin{abstract}
We present a new study on the phenomenon of Urban Heat Island in the Mallorcan city of Inca, with the aim of expanding and verifying the results of previous works. Using the mobile transect method, measurements were carried out in four seasons of the meteorological year: winter, autumn, summer and spring. The results confirm nocturnal heat islands that show temperature differences of up to $5.7^{\circ} \mathrm{C}$-autumn- between the central sector of the city and its rural surroundings. The urban anthropogenic activity in Inca, the reduced width of certain streets, the height buildings, the shortage of vegetation cover and the atmospheric stability, are combined factors that play in favor of this differential thermal increase, apparently more pronounced in cold ambient conditions than in hot conditions. The results are useful for urban planning works with environmental sustainability criteria.
\end{abstract}

Keywords: Urban heat island, urban climate, measuring transects, Majorca, Inca.

\section{Introducción}

Es conocido que las condiciones climáticas en el interior de las ciudades están influenciadas por la emisión de calor antropogénico y por la artificialización del medio que conlleva todo proceso de urbanización. La desnaturalización de los ecosistemas y el reemplazo de los espacios abiertos por edificios y pavimentos asfálticos, hace que los entornos urbanos se comporten como espacios térmicamente más cálidos respecto de su entorno rural. Esta circunstancia permite hablar de un clima específicamente urbano (Oke, 1973; Landsberg, 1981; Moreno, 1993). El fenómeno que mejor lo representa es el de la isla de calor urbana, principalmente en su variante nocturna. Ésta se empieza a producir poco después de la puesta de sol, cuando la temperatura del aire ha empezado a disminuir. La disminución es más rápida en las áreas rurales que en las urbanas, que tienden a conservar en su interior, durante la noche, el calor acumulado durante el día y el generado internamente por el tráfico motorizado, los sistemas domésticos de calefacción y refrigeración o las actividades industriales, entre otros factores antrópicos. En la creación y desarrollo de la isla de calor también influye la geometría urbana, los materiales de construcción, los pavimentos asfálticos, la posición geográfica del lugar y las condiciones meteorológicas imperantes. 
Estos agentes modificadores de las condiciones atmosféricas normales para formar islas urbanas de calor, han sido verificados en multitud de casos en el mundo, desde que las investigaciones sobre el clima urbano comenzaran a desarrollarse en la década de los años 60 del pasado siglo XX, aplicadas sobre todo a ciudades centroeuropeas. Desde entonces, el estudio del fenómeno cuenta con una amplio y variado repertorio de contribuciones científicas. En España se inician a partir de los años 80, con trabajos pioneros como los de López-Gómez y Fernández-García (1984) para la ciudad de Madrid, los de Cuadrat, de la Riva, López y Martí (1993a) para la ciudad de Zaragoza, o los de Moreno (1994) para la de Barcelona, entre otros. Hoy, muchas otras ciudades españolas han sido prospectadas para identificar en ellas el fenómeno, incluso aquellas de medio o pequeño tamaño, como hizo Goikoetxea (1991) para San Sebastián; Dorta, Marzol y Rodríguez (1992) para Santa Cruz de Tenerife; Brunet (1992) para Tarragona; Cuadrat, de la Riva, López y Martí (1993b) para Teruel; o Martínez (2014) para Alicante, entre otros. Algunas de las principales contribuciones adoptan un carácter bioclimatológico y se ocupan de relacionar isla de calor y confort térmico, como hacen Tejedor et al. (2015) para la ciudad de Zaragoza, o Allende, Fernández-García, Rasilla y Alcaide (2018) para la ciudad de Madrid, con la vista puesta en presentar avances para el planeamiento térmico de las áreas urbanas. Dentro del ámbito mediterráneo, destacan las contribuciones de Papanastasiou y Kittas (2012) para el caso de Volos (Grecia), de Atak et al. (2019) para el caso de la ciudad costera de Kyrenia (Chipre), de Martinelli, Kolokotsa y Fiorito (2020) para Bari (Italia), o de Ünal et al. (2020) para Estambul (Turquía). A un nivel europeo e internacional resultan de gran interés las contribuciones que vinculan las diferencias térmicas con los tipos y usos del suelo urbano (Buykantuyev y Wu, 2010; Dienst, Lindén y Esper, 2018). También son de interés los trabajos sobre la influencia e impacto de la vegetación en las variaciones de la intensidad de la isla de calor (Al-Saadi et al., 2020; Morabito et al., 2021).

Las Islas Baleares no han quedado al margen del interés por el estudio de la isla de calor, y el elenco de publicaciones cubre hasta hoy las ciudades de Palma (Alomar-Garau y Llop-Garau, 2018), Ibiza (Serra, 2007) e Inca (Troya, 2008, 2010 y 2011), a las que se unen algunas poblaciones rurales mallorquinas de menor entidad como Llucmajor, Campos, Pollença y Sa Pobla (Troya y Capó, 2012). En el presente trabajo se aborda nuevamente el estudio de la isla de calor nocturna de Inca, localidad interior de la isla de Mallorca, con el objetivo de sumar nuevas exploraciones y nuevos datos a los que ya se tienen sobre el clima urbano de esta ciudad. Además de esto, se pretende incentivar el estudio del clima urbano de ciudades españolas de tamaño mediano o pequeño. Muchas de ellas son periféricas respecto de los municipios centrales capitalinos, pero en ellas reside -con datos del Atlas estadístico de las áreas urbanas de España, de 2012 (Precedo y Mínguez, 2014) - al menos el 65\% de la población española, dato que pone de manifiesto la importancia del proceso de difusión del crecimiento urbano hacia las periferias, como así ocurre en el caso de Inca. 


\section{2. Área de estudio: Inca, ciudad mediterránea de interior}

La ciudad interior de Inca está situada en la parte centro-occidental de la isla de Mallorca, dentro de la comarca del Raiguer, zona de transición entre el llano central (el Pla) y la cordillera de la Serra de Tramuntana. A escala municipal, la población era en el año 2019 de 33.319 habitantes para una superficie de $58,3 \mathrm{~km}^{2}$ (IBESTAT, 2020). La población estrictamente urbana era de 30.892 habitantes para una ciudad de $2,86 \mathrm{~km}^{2}$ de extensión. Con estos datos, el municipio de Inca constituye el tercero de Mallorca con más densidad de población ( 571,5 habitantes por $\mathrm{km}^{2}$ ), solo por detrás de Palma (2.128,1 hab. $\left./ \mathrm{km}^{2}\right)$ y de Marratxí $\left(686,6 \mathrm{hab} . / \mathrm{km}^{2}\right)$. En cuanto a su topografía, el término municipal presenta dos elevaciones contiguas a unos $3 \mathrm{~km}$ de distancia al nordeste de la ciudad -el Puig d'Inca o de Santa Magdalena, y el Puig de Son Sastre-, con una altura de 304 y 291 msnm respectivamente, según el Mapa Topográfico Nacional a escala 1:25.000. Al norte del núcleo urbano y a poca distancia del mismo puede mencionarse el arco formado por tres elevaciones menores: el Putxet d'en Reure -182 msnm-, el Putxet de cal Senyor Pere -186 msnm- y el Puig de Talares -181 msnm-, bajo el que se ubica la urbanización de s'Ermita. La hidrografía aparece marcada por el paso de algunos torrentes, entre los cuales destaca el de Can Tabou -también llamado torrent de Mandrava-, canalizado, que atraviesa el centro de la ciudad. Con datos del Sistema de Información sobre Ocupación del Suelo de España (SIOSE, 2014), en el municipio predominan las áreas de cultivo $(74,8 \%)$, y dentro de éstas los frutales no cítricos $(29,6 \%)$ y los cultivos herbáceos $(14,4 \%)$. Los bosques, en cambio, apenas ocupan el 12,9\% del territorio.

Por otra parte, cuando se lleva a cabo un estudio del clima urbano, es importante considerar si la ciudad examinada es litoral o, por el contrario, se encuentra alejada del mar, ya que el agua del mar, cuyo calor específico es mayor que el del suelo, opera como agente termorregulador que modera las temperaturas en las localidades costeras, y reduce la oscilación térmica diaria entre la máxima diurna y la mínima nocturna. En cambio, en las áreas de interior esta diferencia es mayor. En el caso de Inca, tiene las típicas características de una localización microcontinental porque se encuentra alejada unos $20 \mathrm{~km}$ del mar hacia el noreste (bahía de Alcúdia), y unos $26 \mathrm{~km}$ hacia el suroeste (bahía de Palma). Si bien son distancias que todavía permiten recibir la influencia marítima, esta influencia se elimina por completo en el caso de la distancia al mar por el norte, debido a la pantalla orográfica que representa la Serra de Tramuntana. Con datos del Atlas climático de España para el municipio, la pluviometría es de $569 \mathrm{~mm}$ de promedio anual, mientras que la media anual de temperaturas es de $17,4^{\circ} \mathrm{C}$. La media anual de las mínimas es de $12,5^{\circ} \mathrm{C}$, y de $22,2^{\circ} \mathrm{C}$ la media anual de las máximas. La temperatura media del mes más frío (enero) es de $10,2^{\circ} \mathrm{C}$, mientras que la media del mes más cálido (agosto) es de 26,5ํㅡ. La diferencia entre estas dos últimas medias es de $16,3^{\circ} \mathrm{C}$-en la ciudad litoral de Palma, en cambio, es menor, de $15,2^{\circ} \mathrm{C}$, precisamente porque su localización es marítima-. 
En relación con la geografía urbana, Inca es hoy una ciudad residencial y administrativa con una gran oferta de servicios y negocios, lo que se ha traducido en un exponencial crecimiento poblacional y urbanístico a lo largo de los últimos cien años. Una parte del casco urbano central ocupa la pequeña colina que se extiende de noroeste (Serral de Ses Monges) a sureste. El centro histórico, sobre todo el representado por la barriada de Sant Francesc, presenta una destacable densidad edificatoria, con calles estrechas e irregulares, flanqueadas en general por casas bajas de tipología tradicional de entre dos y tres plantas. Esta composición aparece muy puntualmente modificada por la presencia de ciertos edificios plurifamiliares que llegan a alcanzar diez plantas.

Aparte del centro histórico, forman parte del ensanche urbano de Inca los barrios de Crist Rei y Crist Rei Nou -al suroeste y de planta cuadricular-, Es Blanquer al nordeste y Son Amonda al norte, zonas donde predominan los edificios unifamiliares y plurifamiliares. Son parte caracterizadora de la ciudad las zonas de la Gran Via de Colom y la de Reis Catòlics, ambas con edificaciones en general uniformes, y que dan nombre a dos respectivas avenidas que flanquean por abajo y por arriba el núcleo antiguo. Al sur se encuentra la zona del polígono industrial, formado por grandes naves y vías de circulación más anchas. Así descrito, el aspecto de Inca es el de una ciudad compacta con una relativa carencia de verde urbano en su interior, y con cierta densidad de tránsito motorizado, favorecida por la actividad comercial, principalmente en la Gran Via de Colom, la carretera de Selva, la avenida de Alcúdia y la avenida de Reis Catòlics.

\section{Metodología}

El estudio de isla de calor nocturna de Inca se ha acometido a partir de la toma itinerante de la temperatura del aire a lo largo de un recorrido por las vías de circulación y plazas de la ciudad, y su periferia rural. Este recorrido ha sido planificado y diseñado de tal manera que los puntos de medición cubren todos los barrios, en especial aquellos con más diferencias fisiográficas, cosa que ha permitido obtener mapas térmicos de la ciudad. Se ha seguido, por lo tanto, el conocido método de los transectos móviles urbanos, en este caso con un automóvil en la parte exterior del cual se ha acoplado el sensor externo de un termohigrómetro digital Zelsio P330, con una resolución de $0,1^{\circ} \mathrm{C}$ para la temperatura, y del 0,1\% para la humedad atmosférica. En relación con el método escogido, es el mismo aplicado en multitud de trabajos orientados a identificar islas de calor, aunque autores como Romero et al. (2020) han propuesto recientemente un método alternativo consistente en el diseño que transectos móviles óptimos y representativos, que no dependen de un mero recorrido formal a través de zonas con diferentes características físicas. Su propuesta fue testada en el centro histórico de la ciudad de Sevilla.

Las mediciones se han hecho en cuatro fechas diferentes, que se corresponden con las cuatro estaciones del año meteorológico: 7 de diciembre del 2019 (invierno), 11 de 
marzo de 2020 (primavera), 26 de agosto de 2020 (verano) y 28 de octubre de 2020 (otoño). Ya que el efecto de isla de calor se empieza a notar, sobre todo, poco después de la puesta de sol (Cuadrat, 1994), cuando la temperatura del aire ha empezado a disminuir, las mediciones se han hecho siempre en horario nocturno, y siempre pasadas al menos dos horas después de la puesta. Pese a que conforma una unidad, el recorrido se ha dividido en tres diferentes transectos (Figura 1) con la finalidad de comparar tres distintos escenarios geográficos urbanos. Esto quiere decir que el inicio y el final de cada transecto se ha situado estratégicamente en lugares diferentes en la periferia de la ciudad, y que, además, los transectos confluyen en la zona central de la localidad.

El primer transecto $(5,3 \mathrm{~km}$ de longitud) atraviesa la ciudad de suroeste a nordeste y comprende 46 puntos de medición. Su intersección cartográfica con el mapa de ocupación del suelo del Urban Atlas europeo (Figura 2), de escala 1:15.000, permite ver que los primeros tramos del recorrido urbano cubren suelo industrial, y dentro de él transcurre junto a dos zonas verdes. Una vez en el centro de la ciudad, atraviesa un casco histórico de tejido urbano más o menos denso, y llega hasta una zona abierta con un polideportivo en el extremo nordeste de la ciudad. El segundo transecto (3,9 $\mathrm{km})$ transcurre de sureste a noroeste y comprende 33 puntos de medición. Su recorrido comienza en campo abierto sobre suelo agrícola. Penetra en la ciudad por la avenida Gran Via de Colom -la misma por donde transcurre soterrado el curso hídrico de Can Tabou-, de tejido urbano continuo, hasta alcanzar la barriada occidental de Es Blanquer, zona del ensanche de calles más anchas y rectilíneas, y con una plaza arbolada. El tercer y último transecto $(6,0 \mathrm{~km})$ comprende 49 puntos de medición. Comienza en los aledaños rurales de las barriadas de Crist Rei Nou y Crist Rei, al suroeste de la ciudad, cuyo tejido urbano es discontinuo y poco denso. En esta zona deja a un lado dos zonas verdes, y luego cruza una vía de tren para alcanzar el centro urbano de Inca, de calles más estrechas. Finalmente recorre la calle de Sant Bartomeu hasta alcanzar una zona elevada al norte de la ciudad, en los aledaños del parque verde del Serral, y desde ahí hasta Son Amonda, en el extremo norte, atravesando este barrio de tejido urbano relativamente discontinuo. Unidos los tres transectos, el itinerario totaliza $15,2 \mathrm{~km}$ y 128 puntos de medición. La distribución relativamente regular de esta nube de puntos ha permitido crear interpolaciones de interés y, con ellas, comprobar las morfologías que presenta la isla de calor de Inca en las cuatro sesiones de campo indicadas.

En todos los casos, las mediciones se han hecho en condiciones atmosféricas de estabilidad para evitar aquellas situaciones que típicamente producen interferencias en los registros de temperatura. Así, en la distribución espacial de la temperatura urbana influye no sólo la configuración física de la ciudad -altura y densidad de las edificaciones, trama de las calles, tipo de pavimento- o la presencia y ausencia de vegetación, sino que también influyen ciertos factores meteorológicos como el viento y la nubosidad (Givoni, 1998). Normalmente, los vientos moderados a fuertes modifican a la baja los valores de temperatura urbana, y por tanto hacen que disminuya la intensidad de la isla de calor, como así ocurre, por ejemplo, en los casos estudiados de las ciudades mediterráneas de Barcelona (Moreno, 1993), Roma (Fabrizi, Bonafoni y Biondi, 
2010), Tesalónica (Giannaros y Melas, 2012) o Nicosia (Theophilou y Serghides, 2014). En cuanto a las nubes, si se presentan más o menos compactas retardan el enfriamiento nocturno del aire y la isla de calor no se manifiesta con la eficiencia esperada, pues una excesiva cobertura nubosa produce un efecto de invernáculo en virtud del cual la pérdida de radiación por la superficie terrestre durante la noche, enfriando el suelo y el aire en contacto con él, es en parte compensada por el rebote de la radiación contra las nubes. Ésta retorna hacia la superficie y, en consecuencia, frena el enfriamiento natural de la misma. En el caso del presente trabajo, la toma de datos de la noche del 7 de diciembre de 2019 se hizo con condiciones óptimas de anticiclón, con altas presiones (1023 hPa) sobre las Baleares, cielos despejados en Inca y vientos flojos o en calma. Muy parecidas condiciones se dieron la noche del 11 de marzo de 2020, dominada por las altas presiones $(1020 \mathrm{hPa})$, vientos en calma o flojos del suroeste, y sin nubosidad. También la noche del 26 de agosto de 2020 fue de estabilidad atmosférica, cielos despejados y vientos flojos del sur y suroeste. La noche del 28 de octubre de 2020 fue también despejada, con vientos flojos del nordeste.

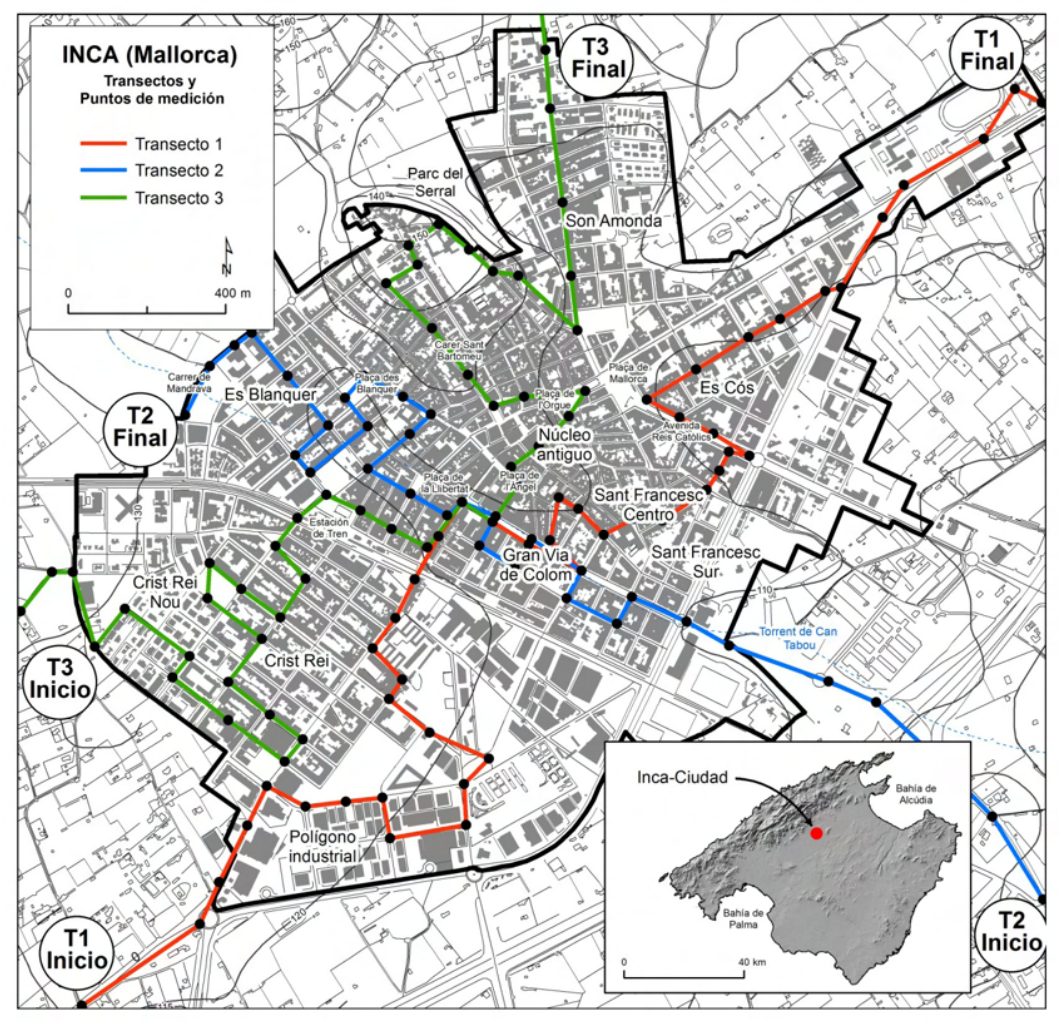

Figura 1. Recorrido y numeración de los transectos por la ciudad de Inca y sus barriadas.

Fuente: Elaboración propia. 


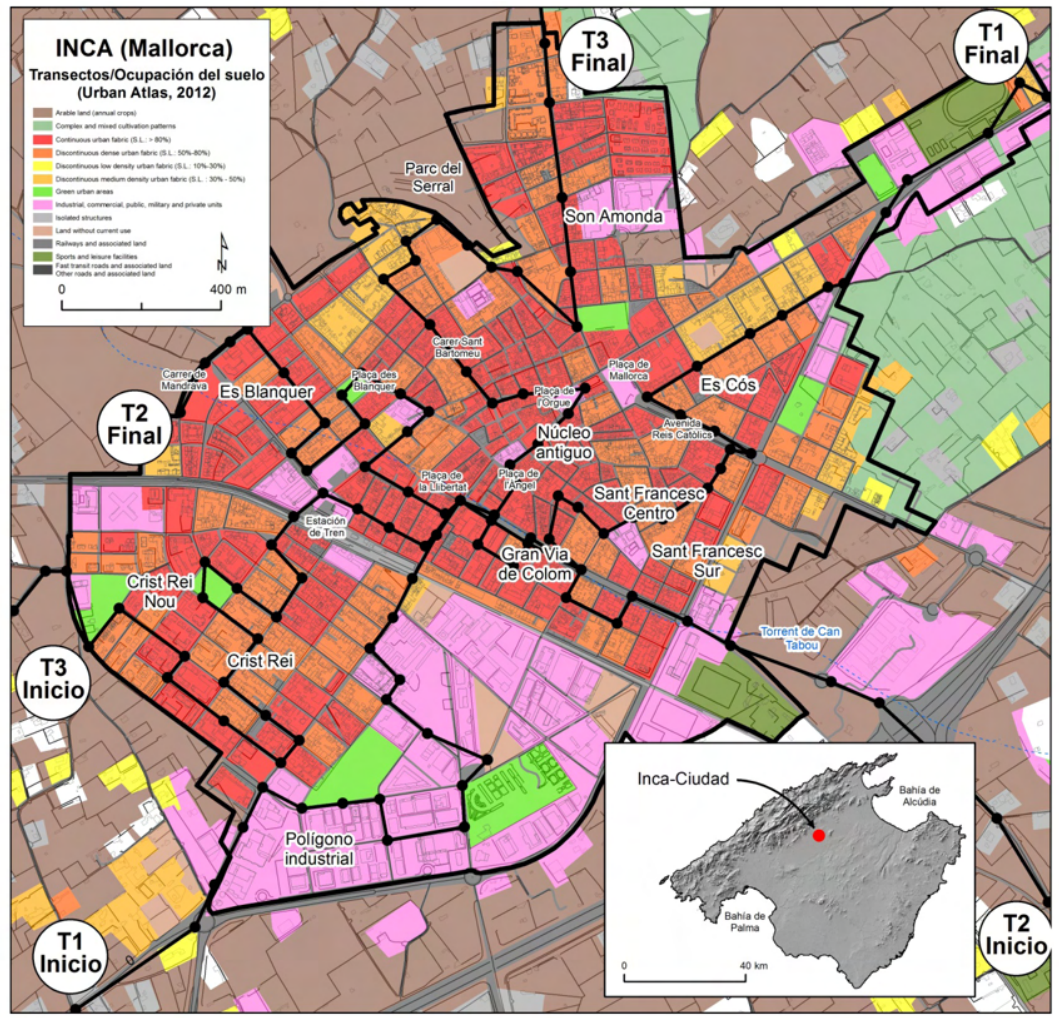

Figura 2. Recorrido y ocupación del suelo en Inca (Urban Atlas, 2012).

Fuente: Elaboración propia.

\section{Resultados y discusión}

De acuerdo con las mediciones obtenidas en aplicación del método descrito, los resultados confirman la presencia de una isla de calor morfológicamente agrupada, en general, en la zona central de la ciudad. Esto se consigna para cada una de las cuatro mediciones de campo y para cada uno de los cuatro transectos considerados, a pesar de los matices que a continuación se especifican. La máxima intensidad de isla de calor registrada es de $5,7^{\circ} \mathrm{C}$ y se alcanza la noche del 28 de octubre de 2020 , es decir en el otoño meteorológico (Tabla 1). Este valor se considera de intensidad fuerte, si seguimos el extendido criterio de Fernández-García (1996) según el cual la intensidad es débil cuando la diferencia es de hasta $2^{\circ} \mathrm{C}$, moderada cuando es de $2^{\circ}$ a $4^{\circ} \mathrm{C}$, fuerte cuando es de $4^{\circ}$ a $6^{\circ} \mathrm{C}$, y muy fuerte cuando supera los $6^{\circ} \mathrm{C}$. Además de esto, se trata de un valor que supera el que se esperaría para una ciudad como Inca aplicando la fórmula de Oke (1973 
y 1987), diseñada para calcular la intensidad esperable de isla de calor en función de su población. Para una población urbana en Inca de 30.892 habitantes (año 2019), la fórmula da un valor de intensidad máxima esperable de 4,9 $\mathrm{C}$. El segundo registro de máxima intensidad corresponde a la noche del 7 de diciembre de 2019 , con 4,2을 $\mathrm{C}$. En marzo y agosto se consignan intensidades de $1,5^{\circ} \mathrm{C}$ y $3,0^{\circ} \mathrm{C}$, respectivamente.

Tabla 1. Resumen de resultados de la intensidad de la isla de calor urbana (ICU) en Inca, y valores térmicos máximos y mínimos registrados durante los recorridos

\begin{tabular}{|l|c|c|c|c|c|c|c|}
\hline Fecha & Transecto & $\begin{array}{c}\text { Hora de } \\
\text { inicio }\end{array}$ & \multicolumn{2}{|c|}{$T^{\underline{a}}$ máx. $\left({ }^{\circ} \mathrm{C}\right)$} & \multicolumn{2}{|c|}{$T^{\underline{a}}$ mín. $\left({ }^{\circ} \mathrm{C}\right)$} & \multicolumn{1}{c|}{$\begin{array}{c}\text { Intensidad } \\
\text { máx. ICU }\left({ }^{\circ} \mathrm{C}\right)\end{array}$} \\
\hline 7 diciembre 2019 & Transecto 1 & $20: 45$ & 11,5 & & 8,3 & & \\
(invierno) & Transecto 2 & $21: 05$ & 11,1 & 11,5 & 7,3 & 7,3 & $\mathbf{4 , 2}$ \\
& Transecto 3 & $21: 25$ & 11,1 & & 7,5 & & \\
\hline 11 marzo 2020 & Transecto 1 & $21: 40$ & 14,0 & & 13,1 & & \\
(primavera) & Transecto 2 & $21: 55$ & 13,6 & 14,0 & 12,8 & 12,5 & $\mathbf{1 , 5}$ \\
& Transecto 3 & $22: 15$ & 13,5 & & 12,5 & & \\
\hline 26 agosto 2020 & Transecto 1 & $22: 30$ & 27,3 & & 25,1 & & \\
(verano) & Transecto 2 & $22: 50$ & 26,5 & 27,3 & 24,9 & 24,3 & $\mathbf{3 , 0}$ \\
& Transecto 3 & $23: 10$ & 26,9 & & 24,3 & & \\
\hline 28 octubre 2020 & Transecto 1 & $21: 50$ & 17,7 & & 13,8 & & \\
(otoño) & Transecto 2 & $22: 10$ & 16,4 & 17,7 & 12,2 & 12,0 & $\mathbf{5 , 7}$ \\
& Transecto 3 & $22: 25$ & 15,7 & & 12,0 & & \\
\hline
\end{tabular}

Fuente: Elaboración propia.

Estos resultados primarios sugieren que la intensidad de la isla de calor parece mayor en condiciones meteorológicas de frío ambiental, y es menor en condiciones de más calor. Esto resulta coherente con la hipótesis según la cual los días más fríos del año son más propicios para el establecimiento de una isla de calor típica (Moreno, 1994; Montávez, Rodríguez y Jiménez, 2000), ya que aumenta la combustión producida por las calefacciones de uso doméstico y aumenta, en general, la actividad combustiva urbana, lo que se traduce en un incremento de la temperatura ambiental de la ciudad respecto de su periferia rural. Aunque no hay un consenso claro en torno a la estacionalidad de la isla de calor, otros autores señalan que puede ser más intensa en verano (Kolokotroni y Giridharan, 2008; Klysik y Fortuniak, 1999).

Ya que el presente trabajo pretende ampliar el estudio de la isla de calor de Inca y sumarse a los estudios precedentes, es oportuno referirse a los resultados obtenidos en estos otros estudios y establecer comparaciones de interés. En un primer trabajo de 
Troya, publicado en 2008 aunque con datos del año 2007, se consignan unos valores de isla de calor nocturna de hasta $5,3^{\circ} \mathrm{C}$, que es la diferencia entre el punto más frío medido en la zona periférica de Mandrava -al noroeste de la ciudad- y el punto más cálido medido en la Plaça de la Llibertat, el día 1 de febrero de 2007. Ese mismo valor lo obtiene Troya (2008 y 2010) el día 20 de abril de 2007, con la diferencia según la cual los puntos adicionalmente más cálidos de la ciudad se localizaban en la Gran Via de Colom, la Plaça de l'Àngel y la Plaça de l'Orgue. En el caso del presente trabajo, la mínima temperatura de la noche del 7 de diciembre de $2019-7,3{ }^{\circ} \mathrm{C}-$ también se obtiene en la calle periférica de Mandrava, mientras que las máximas -11,1 y 11,5 ${ }^{\circ} \mathrm{C}$ - se obtienen en la avenida Reis Catòlics (Es Cós), en la Gran Via de Colom y en general en la barriada de Sant Francesc Centro (Figura 3a).

La noche del 28 de octubre de 2020 , mínimas temperaturas entre $12,0^{\circ}$ y $13,8^{\circ} \mathrm{C}$ se obtienen, lógicamente, en el ámbito rural, así como también en la calle de Mandrava (Figura 3d). La máxima de $17,7^{\circ} \mathrm{C}$ lo hace en la Gran Vía de Colom, pero otras máximas se generalizan en las áreas más meridionales de Crist Rei y del polígono industrial, por lo que la isla de calor, aunque su foco está en el casco histórico y en Gran Via de Colom, aparece desplazada hacia el sur. Las mínimas de $24,3^{\circ} \mathrm{C}$ de la noche del 26 de agosto de 2020 aparecen al oeste de la ciudad, muy cercanas a la barriada periférica de Crist Rei Nou, mientras que las máximas en torno a los $27,3^{\circ} \mathrm{C}$ lo hacen en el casco urbano histórico y en Gran Via de Colom (Figura 3c). Lo mismo ocurre en el caso de las mínimas del 11 de marzo de 2020 -12,5ำ-, que aparecen también junto a Crist Rei Nou. En cambio, las máximas de esta noche de primavera $-14,0^{\circ} \mathrm{C}-$ aparecen desplazadas hacia el noreste -avenida Reis Catòlics-, sin menoscabo del centro histórico. Este desplazamiento se produce hacia sotavento en el sentido de la dirección del viento a la hora de la medición, que era del suroeste (Figura 3b).

Por lo demás, si la producción de calor antropogénico se presume que es comparativamente mayor en los meses fríos que en los cálidos de verano, así se traduce en los cuatro casos analizados, en que la isla de calor otoñal e invernal es de intensidad fuerte, mientras que es moderada y débil en verano y primavera, respectivamente. En los trabajos precedentes de Troya (2008 y 2010), en los que se analizan las islas de calor producidas en 23 días diferentes del año, la estación veraniega es la que presenta un mayor número de casos de islas de calor de intensidad débil. Aún así, esto no impide que también en verano se den casos puntuales de intensidades mayores -en el presente trabajo, la de la noche del 26 de agosto fue moderada, de 3,0 $\mathrm{C}-$, lo que significa que la magnitud del fenómeno depende en gran parte de las condiciones ambientales del momento, con cierta independencia de la estación en la que nos encontremos. Por otra parte, resulta interesante comprobar que en ciudades de Mallorca de pequeño tamaño como Llucmajor, con una población urbana, en 2019, de 10.830 habitantes (casi tres veces menor que la de Inca), se identifican islas de calor de hasta 6,7ํㅡ C (Troya y Capó, 2012; Moreno y Serra, 2016). En la ciudad balear de Ibiza, cuya población alcanzó en 2019 los 49.556 habitantes, Serra (2007) consignó islas de calor con intensidades de hasta $6,4^{\circ} \mathrm{C}$. 

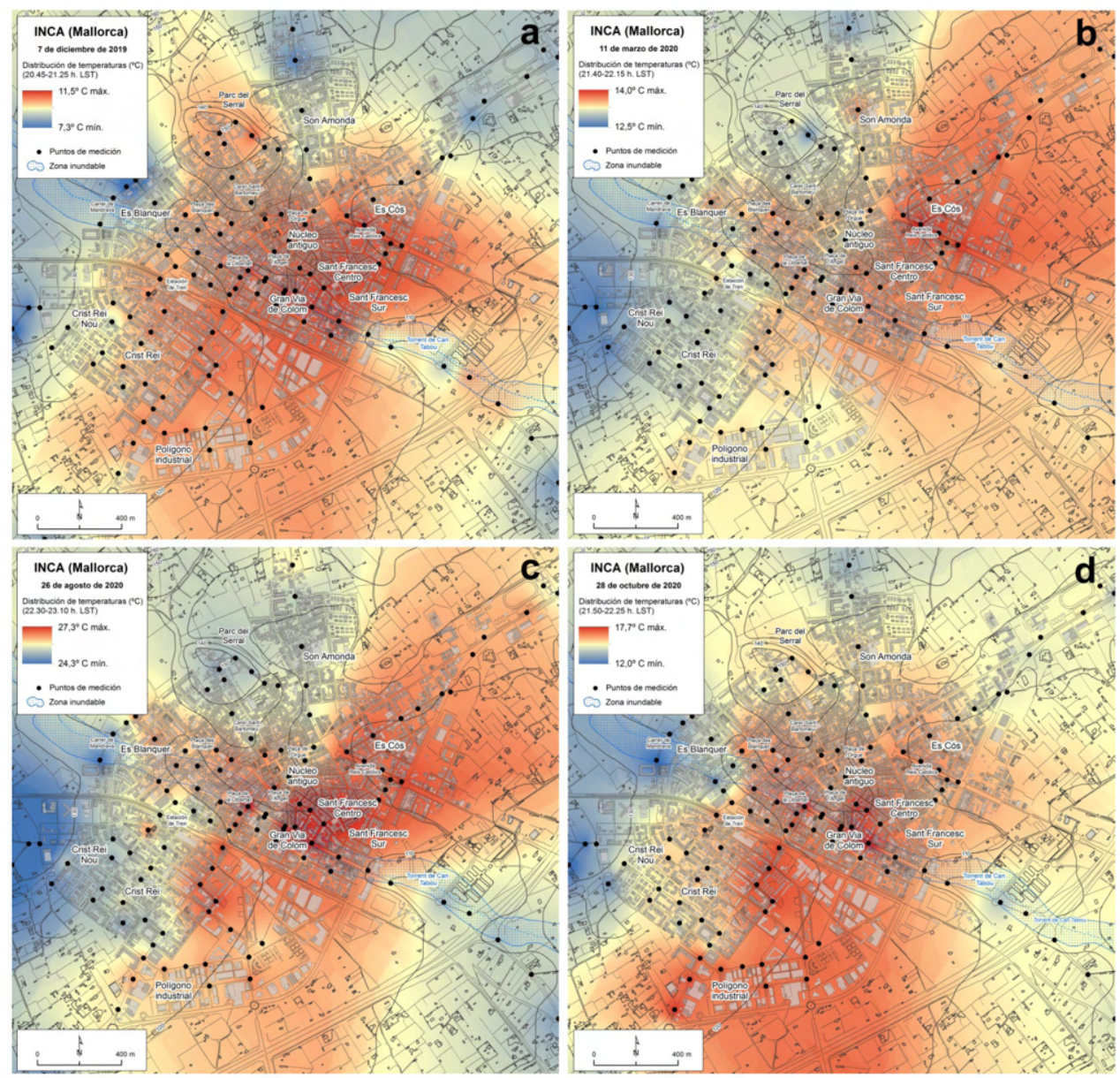

Figura 3. Morfologías de la isla de calor urbana los días 7 de diciembre de 2019 (a), 11 de marzo de 2020 (b), 26 de agosto de 2020 (c) y 28 de octubre de 2020 (d).

Fuente: Elaboración propia.

Para conocer las variaciones y evolución de la temperatura a lo largo de los transectos urbanos realizados en Inca, es útil recurrir a los perfiles térmicos de las gráficas de la Figura 4. Éstas muestran la variación de la intensidad de la isla de calor urbano desde el inicio del transecto hasta su final. En las gráficas, los datos de temperatura se han traducido a valores absolutos, reduciendo a 0 el valor de temperatura del punto de inicio de cada recorrido. En todos los casos se aprecia la formación de una isla de calor típica, con distintos grados de regularidad morfológica. Los tres perfiles térmicos muestran que la intensidad de la isla de calor ha sido mayor en las jornadas nocturnas de otoño e invierno que en las de verano y primavera. 
Las causas que provocan una distribución espacial de temperaturas como la representada hay que encontrarlas, en primer lugar, en las diferentes coberturas de suelo por las que se desplaza el sensor: las coberturas con suelos naturales, con láminas de agua y con vegetación se enfrían con más rapidez que las coberturas artificiales de asfalto y sin cubierta vegetal, que retienen calor. Esto se confirma, sobre todo, en los perfiles de los transectos 1 y 3 , en que al inicio de los recorridos, la transición térmica entre el campo y la ciudad se produce de manera abrupta - con la única excepción de la noche de verano-, precisamente porque los cambios del tipo de superficie-de suelos de tierra y con vegetación a pavimentos asfaltados- tienen un reflejo inmediato en la temperatura ambiental. En segundo lugar, la intensidad de la isla de calor, una vez formada en el interior de la ciudad, varía en función de las diferentes características viarias y edificatorias, y de las variaciones geomórficas del terreno. Así, flanqueadas por edificios de cierta altura, las calles estrechas, de corto recorrido y con menor ventilación natural ven incrementada su temperatura ambiental durante la noche porque impiden una pérdida eficaz del calor acumulado durante el día, que queda atrapado en el interior de la calle (Eliasson, 1994). Esto, incluso en unas calles que tienen poco tráfico rodado. Esta circunstancia hace que las zonas del casco urbano histórico no se enfríen tan rápidamente como los espacios abiertos, presentándose más cálidas de lo que correspondería sin el añadido de la urbanización. El mismo efecto se detecta en cascos históricos de otras ciudades de Mallorca, de factura medieval, como así lo observan Alomar-Garau y Llop-Garau (2018) en ciertas barriadas del antiguo casco intramuros de la ciudad de Palma. Troya y Capó (2012) lo confirman también en el caso de poblaciones mallorquinas de menor entidad como Campos o Pollença.

La mayor capacidad calorífica de unos núcleos urbanos de estas características explica que tanto en el núcleo antiguo de Inca como en el inmediato Sant Francesc, incluyendo la Gran Via de Colom, se consignen las máximas intensidades de isla de calor -calle Miquel Mir, calle Son Net, calle Sant Miquel, calle del Vent-. En cambio, las barriadas de Crist Rei y Crist Rei Nou, a pesar de que son zonas urbanizadas y que soportan cierto tráfico motorizado, se comportan térmicamente como áreas no tan cálidas, porque la densidad de las edificaciones, en general unifamiliares y plurifamiliares con la ocasional presencia de jardines domésticos, es mucho menor que la que se puede encontrar en el núcleo antiguo. Los valores que hemos obtenido en el sector central de Crist Rei contrastan con los que presenta Troya (2011) en los mapas de isotermas invernales de su memoria del proyecto de investigación del Programa de Doctorado en Geografía, de la Universitat de les Illes Balears. En algunos de estos mapas el autor señala en Crist Rei una zona secundaria de isla de calor, seguramente a causa del desplazamiento de la isla a sotavento de la dirección predominante del viento, en esos casos del nordeste. En nuestro trabajo, este efecto se identifica con claridad en la noche otoñal del 28 de octubre de 2020, en que toda la zona de sur de Inca, en la que se incluye su polígono industrial -calle Sabaters, calle Fusters-, aparece casi tan cálida como la zona central. Otra de las zonas de Inca comparativamente más cálidas es la de la avenida Reis Catòlics, circunstancia que se explica por ser una vía que soporta 

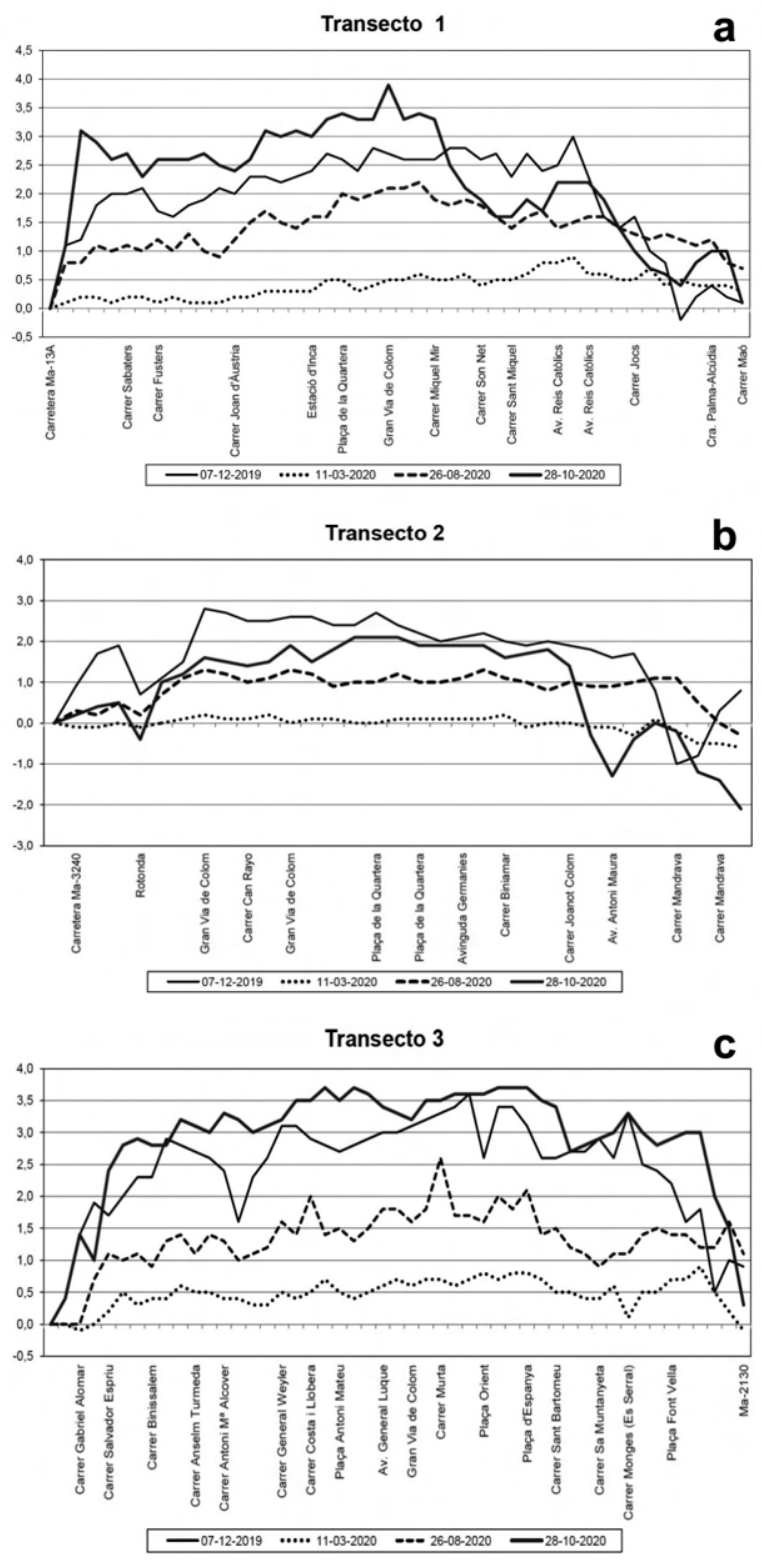

Figura 4. Perfiles térmicos de los transectos 1,2 y 3 ( $a$, b y c). Los ejes de la izquierda muestran el rango de valores de aumento y disminución de temperatura $\left({ }^{\circ} \mathrm{C}\right)$ relativos al valor reducido a 0 del inicio de los transectos. 
una gran actividad comercial y donde el tráfico de vehículos a motor es relativamente intenso. La cobertura arbórea en esta avenida parece que no tiene la influencia esperada a la hora de reducir las temperaturas nocturnas de la zona.

En el lado contrario de estas situaciones de calor agregado se encuentran las barriadas más periféricas y modernas del ensanche urbano de Inca, sobre todo Son Amonda, en el sector septentrional, y Es Molins o Es Blanquer, barriadas más occidentales. Es Blanquer se beneficia de unas temperaturas relativamente menos cálidas gracias, probablemente, a la influencia topográfica que supone el paso del curso hídrico del torrent de Can Tabou, pero también al hecho que las calles son más anchas y los edificios son en general de dos plantas o una, y algunos con corral. Si las vaguadas de los torrents tienen el efecto de reducir la temperatura ambiental por acumulación de aire frío, esto no parece que ocurra en el caso del curso hídrico que transcurre soterrado por la Gran Via de Colom. Esta avenida se comporta como una zona claramente cálida, de forma que ni la eventual influencia del agua en el subsuelo ni el arbolado viario parecen suficientes para corregir a la baja los efectos de la isla de calor. Igualmente, la escasez de zonas verdes de suficiente amplitud en el interior de Inca es un factor que juega en detrimento de las posibilidades de mitigación de los efectos de la isla de calor (Givoni, 1989; Bass, 2003; Rosenzweig et al., 2006; Melaas, Wang, Miller y Friedl, 2016).

\section{Conclusiones}

A partir de una campaña de mediciones nocturnas de la temperatura realizadas en la ciudad mallorquina de Inca entre 2019 y 2020, hemos verificado la presencia de islas de calor típicas en las cuatro noches testadas, correspondientes a cuatro diferentes estaciones del año. Se trata de una nueva contribución al estudio del fenómeno para ciudades de clima mediterráneo de mediano o pequeño tamaño, cuyo interés radica en comprobar cómo los ambientes urbanos, bajo determinadas condiciones atmosféricas y aunque el volumen urbano sea reducido, generan sectores de calor adicional a tener en cuenta cuando se acomete la planificación de la ciudad. En el caso de Inca, la compacidad del casco urbanizado, la densidad edificatoria y el calor antropogénico, son factores que juegan a favor de la creación de islas de calor, con intensidades moderadas o fuertes, pues se superan los $5^{\circ} \mathrm{C}$ de diferencia entre la ciudad y su entorno rural. La formación de burbujas urbanas diferencialmente más cálidas se produce, sobre todo, en el casco histórico de mayor densidad edificatoria y de calles más estrechas -barriada de Sant Francesc-, además de las dos principales avenidas, Gran Via de Colom y Reis Catòlics, precisamente las que soportan más tráfico rodado y actividad comercial. Las barriadas limítrofes del ensanche noroccidental (Es Blanquer, Son Amonda), así como las de Crist Rei, se ven menos afectadas por el fenómeno, de manera que allí el enfriamiento nocturno resulta más eficaz. No obstante, si bien las islas de calor observadas ocupan una posición más o menos central en la ciudad, no es raro que la burbuja cálida se desplace a sotavento de la dirección del viento imperante. 
Las intensidades de isla de calor consignadas oscilan entre los $1,5^{\circ} \mathrm{C}$ y $3,0^{\circ} \mathrm{C}$ de marzo y agosto, respectivamente, y los $4,2^{\circ} \mathrm{C}$ y $5,7^{\circ} \mathrm{C}$ de diciembre y octubre. Esto parece indicar que el fenómeno es más intenso cuando se presentan condiciones atmosféricas de frío, mientras que se debilita en condiciones de relativo calor. Sin embargo, las cuatro noches examinadas, aunque se han hecho corresponder con las cuatro estaciones del año, no son suficientes para extraer de sus resultados unas conclusiones categóricas, entre ellas que la intensidad de la isla de calor es mayor en invierno u otoño, y menor en verano o primavera. Para comprobarlo haría falta extender la campaña de mediciones a una escala temporal mensual.

Por otra parte, el método de los transectos urbanos utilizado en el trabajo, así como el diseño de los recorridos, específico para la ocasión (tres itinerarios en total con un total de 128 puntos de medición), se han mostrado plenamente adecuados, y deben servir en futuras campañas que permitan comprobar la evolución del fenómeno a escalas temporales más amplias, aquellas con las que se puedan observar los presumibles efectos del calentamiento global y del cambio climático. A largo plazo, estos efectos se espera que tengan un amplio impacto en los ecosistemas urbanos, que experimentarán cambios estructurales a los que se hace necesario anticiparse. Una de las claves se encuentra en la mejora y desarrollo de zonas verdes y de arbolado viario en el interior de la ciudad, por el papel determinante de la vegetación en la refrigeración de la ciudad cuando está sometida a situaciones meteorológicas de calor.

\section{Bibliografía}

Al-Saadi, L.M., Jaber, S.H. y Al-Jiboori, M.H. (2020). Variation of urban vegetation cover and its impact on minimum and maximum heat islands. Urban Climate 34, 100707. DOI: https:// doi.org/10.1016/j.uclim.2020.100707

Allende, F., Fernández-García, F., Rasilla, D. y Alcaide, J. (2018). Isla de calor nocturna estival y confort térmico en Madrid: avance para un planeamiento térmico en áreas urbanas. Ciudad y Territorio, Estudios Territoriales (CyTET) 50 (195), pp. 101-120.

Alomar-Garau, G. y Llop-Garau, J. (2018). La isla de calor urbana de Palma (Mallorca, Islas Baleares): avance para el estudio del clima urbano en una ciudad litoral mediterránea. Boletín de la Asociación Española de Geografía 78, pp. 392-418. DOI: http://dx.doi.org/10. 21138/bage. 2404

Atak, M., Kara, C., Buket, A. y Özden, O. (2019). The urban heat island in a coastal Mediterranean city: The case study of Kyrenia, Cyprus. International Journal of Advanced and Applied Sciences 6 (8), pp. 1-8. DOI: http://dx.doi.org/10.21833/ijaas.2019.08.001

Bass, B. y Baskaran, B. (2003). Evaluating Rooftop and Vertical Gardens as an Adaptation Strategy for Urban Areas. Ottawa (Canada): National Research Council Canada, Institute for Research in Construction. Report no. NRCC-46737.

Brunet, M. (1992). La magnitud y fluctuaciones de la isla de calor de Tarragona. Tarraco 7, pp. 19-29. 
Buykantuyev, A. y Wu, J. (2010). Urban heat islands and landscape heterogeneity: linking spatiotemporal variations in surface temperatures to land-cover and socioeconomic patterns. Landscape Ecology 25 (1), pp. 17-33. DOI: https://doi.org/10.1007/s10980-009-9402-4

Cuadrat, J.M., de la Riva, J., López, F. y Martí, A. (1993a). El medio ambiente urbano en Zaragoza. Observaciones sobre la "isla de calor". Anales de Geografía de La Universidad Complutense 13, pp. $127-138$.

Cuadrat, J.M., de la Riva, J., López, F. y Martí, A. (1993b). Ciudad y Medio Ambiente: la isla de calor de Teruel. GEOGRAPHICALIA 30, pp. 113-123.

Cuadrat, J.M. (1994). “Los climas urbanos en el Valle del Ebro». En: López-Gómez, A. (coord) El clima de las ciudades españolas. Madrid: Cátedra.

Dienst, M., Lindén, J. y Esper, J. (2018). Determination of the urban heat island intensity in villages and its connection to land cover in three European climate zones. Clim. Res. 76, pp. 1-15. DOI: https://doi.org/10.3354/cr01522

Dorta, P., Marzol, M.V. y Rodríguez, J. (1992). Estudio del clima urbano en una ciudad litoral. El caso de Santa Cruz de Tenerife (Islas Canarias). En: VI Trobades Científiques a la Mediterrània. Energia, medi ambient i edificació. (pp. 69-89). Barcelona: CIRIT, Generalitat de Catalunya.

Eliasson, I. (1994). Urban-suburban-rural air temperature differences related to street geometry. Physical Geography 15, pp. 1-22.

Fabrizi, R., Bonafoni, S. y Biondi, R. (2010). Satellite and Ground-Based Sensors for the Urban Heat Island Analysis in the City of Rome. Remote Sensing 2 (5), pp. 1400-1415. DOI: https:// doi.org/10.3390/rs2051400

Fernández-García, F. (1996). Manual de climatología aplicada. Clima, medio ambiente y planificación. Madrid: Editorial Síntesis.

Giannaros, T.M. y Melas, D. (2012). Study of the urban heat island in a coastal Mediterranean City: The case study of Thessaloniki, Greece. Atmospheric Research 118, pp. 103-120. DOI: https:// doi.org/10.1016/j.atmosres.2012.06.006

Givoni, B. (1989). Urban design for different climates (Report WMO-TD, 346). World Meteorological Organization.

Givoni, B. (1998). Climate considerations in building and urban design. USA: John Wiley \& Sons, Inc.

Goikoetxea, I. (1991). El medio ambiente urbano en Donostia. Observaciones sobre la isla de calor generada en el medio urbano. Lurralde 14, pp. 43-162.

Klysik, K. y Fortuniak, K. (1999). Temporal and spatial characteristics of urban heat island of Lòdz, Poland. Atmospheric Environment 33, pp. 3885-3895.

Kolokotroni, M. y Giridharan R. (2008). Urban heat island intensity in London: An investigation of the impact of physical characteristics on changes in outdoor air temperature during summer. Solar Energy 82 (11), pp. 986-998. DOI: https://doi.org/10.1016/j.solener.2008.05.004

Landsberg, H.E. (1981). The urban climate. New York: Academic Press.

López-Gómez, A. y Fernández-García, F. (1984). La isla de calor en Madrid: avance de un estudio de clima urbano. Estudios Geográficos 45 (174), pp. 5-34. 
Martinelli, A., Kolokotsa, D-D. y Fiorito, F. (2020). Urban Heat Island in Mediterranean Coastal Cities: The Case of Bari (Italy). Climate 8 (6), p. 79. DOI: https://doi.org/10.3390/cli8060079

Martínez, J. (2014). Estudio de la isla de calor de la ciudad de Alicante. Investigaciones Geográficas 62, pp. 83-99. DOI: http://dx.doi.org/10.14198/INGEO2014.62.06

Melaas, E.K., Wang, J.A., Miller, D.L. y Friedl, M.A. (2016). Interactions between urban vegetation and surface urban heat islands: A case study in the Boston metropolitan region. Environmental Research Letters 11 (5), 054020.

Montávez, J.P., Rodríguez, A. y Jiménez, J.L. (2000). A study of the urban heat island of Granada. Int. J. Climatol. 20, pp. 899-911.

Morabito, M., Crisci, A., Guerri, G., Messeri, A., Congedo, L. y Munafò, M. (2021). Surface urban heat islands in Italian metropolitan cities: Tree cover and impervious surface influences. Science of the Total Environment 751, 142334. DOI: https://doi.org/10.1016/j.scitotenv. 2020.142334

Moreno García, M.C. (1993). Estudio del clima urbano de Barcelona: la "isla de calor" Barcelona: Editorial Oikos-Tau.

Moreno García, M.C. (1994). Intensity and form of the urban heat island in Barcelona. International Journal of Climatology 6, pp. 705-710. DOI: https://doi.org/10.1002/joc.3370140609

Moreno García, M.C. y Serra Pardo, J.A. (2016). El estudio de la isla de calor urbana en el ámbito mediterráneo: una revisión bibliográfica. Biblio 3 W. Revista Bibliográfica de Geografía y Ciencias Sociales Vol. XXI (1), pp. 1-32.

Oke, T.R. (1973). City size and the urban heat island. Atmospheric Environment 7, pp. 769-779.

Oke, T.R. (1987). Boundary Layer Climates. London: Routledge.

Papanastasiou, D.K. y Kittas, C. (2012). Maximum urban heat island intensity in a medium-sized coastal Mediterranean city. Theoretical and Applied Climatology 107 (3-4), pp. 407-416. DOI: https://doi.org/10.1007/s00704-011-0491-z

Precedo, A. y Mínguez, A. (2014). Una radiografía de las ciudades españolas: una evaluación del modelo postindustrial. En: Lois, R. y Miramontes, A. (eds.) Reflexiones sobre las ciudades y el sistema urbano en tiempos de crisis. (pp. 15-69.). Santiago de Compostela: Universidade de Santiago de Compostela, Grupo ANTE.

Romero, L., Sánchez Ramos, J., Sanchez de la Flor, F.J. y Álvarez, S. (2020). Analyzing the urban heat Island: Comprehensive methodology for data gathering and optimal design of mobile transects. Sustainable Cities and Society 55, 102027. DOI: https://doi.org/10.1016/j.scs. 2020.102027

Rosenzweig, C., Solecki, W., Slosberg, R., Parshall, L., Gaffin, S., Lynn, B., Goldberg, R. y Cox, J. (2006). Mitigating New York City's heat island with urban forestry, living roofs, and light surfaces. A report to the New York State Energy Research and Development Authority.

Serra Pardo, J.A. (2007). Estudio de la isla de calor de la ciudad de Ibiza. Investigaciones Geográficas 44, pp. 55-73.

Tejedor, E., Cuadrat, J.M., Saz, M.A., Serrano, R., López, N. y Aladrén, M. (2015). Islas de calor y confort térmico en Zaragoza durante la ola de calor de julio de 2015. En: Olcina, J., Rico, A.M. y Moltó, E. (eds) Clima, Sociedad, Riesgos y Ordenación del Territorio. (pp. 141-151). Asociación Española de Climatología, Serie A, 10. 
Theophilou, M.K y Serghides, D. (2014). Heat island effect for Nicosia, Cyprus. Advances in Building Energy Research 8 (1), pp. 63-73. DOI: https://doi.org/10.1080/17512549.2014.890538

Troya López, J.A. (2008). L'illa de calor nocturna a Inca. VIIIJornades d'Estudis Locals. (pp. 165-173). Inca: Ajuntament d'Inca.

Troya López, J.A. (2010). Evaluación de la isla de calor nocturna en un núcleo urbano de tamaño mediano: Inca (Mallorca). En: Fernández, F.; Galán, E. y Cañada, R. (eds) VII Congreso de la Asociación Española de Climatología: clima, ciudad y ecosistemas. (pp. 575-586). Barcelona: Serbal. Publicaciones de la Asociación Española de Climatología. Serie A, 7.

Troya López, J.A. (2011). L'illa de calor nocturna a Inca. Memòria del projecte d'investigació del Programa de Doctorat en Geografia de la Universitat de les Illes Balears. Departament de Ciències de la Terra. Tesina de doctorado inédita.

Troya López, J.A. y Capó Esteva, D. (2012). L'illa de calor nocturna a diversos nuclis de població a Mallorca. XIII Coloquio Ibérico de Geografía. Respuestas de la Geografía Ibérica a la crisis actual. (pp. 1175-1187). Santiago de Compostela: Meubook.

Ünal, Y.S., Sonuc, C.Y., Incecik, S., Topcu, H.S., Diren-Üstün, D.H. y TemizÖz, H.P. (2020). Investigating urban heat island intensity in Istanbul. Theoretical and Applied Climatology, 139 (1-2), pp. 175-190. DOI: https://doi.org/10.1007/s00704-019-02953-2 